www.jmscr.igmpublication.org

Impact Factor 5.84

Index Copernicus Value: 83.27

ISSN (e)-2347-176x ISSN (p) 2455-0450

crossref DOI:_https://dx.doi.org/10.18535/jmscr/v5i3.08

Journal Of Medical Science And Clinical Research

\title{
Role of MRI-MRCP in Evaluation of Obstructive Jaundice
}

Authors

\section{Arjun Raju $\mathbf{P}^{1}$, Divyashree ${ }^{2}$, Surabhi Mehrotra ${ }^{3}$}

M.D Radiodiagnosis, Department of Radiodiagnosis,

Government Medical College and Susheela Tiwari Memorial Hospital, Haldwani, Uttarakhand-263139

Email_larjunraju1988@yahoo.com, 2divya.pulakpuneet@gmail.com

surabhimehrotra175@gmail.com

\section{ABSTRACT}

Background- Evaluation of a suspected biliary tract disease is a common surgical problem and traditionally diagnosed by a variety of imaging modalities including Ultrasonography (USG), Computed tomography (CT), Invasive cholangiography and Magnetic Resonance Cholangiopancreatography $(M R C P)$. In a suspected case of biliary obstruction with clinical and laboratory findings suggestive of obstructive jaundice, the main aim of radiologist is to confirm the presence of obstruction and to identify its location, extent and probable cause.

Aim Of Study- To evaluate the diagnostic value of MRCP in studying the sites and causes of obstructive jaundice, and to determine the accuracy of MRCP in the diagnosis of obstructive jaundice.

Materials \& Methods- Twenty patients with clinical \& biochemical features of obstructive jaundice who presented to the department of radiodiagnosis between October 2015 and September 2016 were enrolled in this study. The final diagnosis was found by endoscopic retrograde cholangiopancreatography (ERCP) and lor surgery and confirmed by histopathology.

Results- MRCP was able to diagnose ductal dilatation and hence, differentiate surgical from medical jaundice in all the patients (100\%). MRCP was accurately able to detect the level of obstruction in $100 \%$ of cases. The most common cause of obstructive jaundice was pancreatico-biliary tumors (50\%), followed by choledocholithiasis (40\%). MRCP was accurately able to detect the cause of obstruction in $95 \%$ of cases.

Conclusion- Magnetic resonance imaging - magnetic resonance cholangiopancreatography (MRI-MRCP) is a highly sensitive non-invasive technique in the detection of the presence of biliary obstruction in addition to defining its level and cause. The accuracy of magnetic resonance cholangiopancreatography and its non-invasiveness have considerably restricted the diagnostic role of Endoscopic retrograde cholangiopancreatography (ERCP).

Keywords- Obstructive jaundice, MRI, MRCP. 


\section{INTRODUCTION}

Obstructive jaundice is strictly defined as a condition occurring due to a block in the pathway between the site of conjugation of bile in the liver cells and the entry of bile into the duodenum through the ampulla. The block may be intrahepatic or extrahepatic in the bile duct ${ }^{1,2}$.

In a suspected case of biliary obstruction with clinical and laboratory findings suggestive of obstructive jaundice, the main aim of radiologist is to confirm the presence of obstruction and to identify its location, extent and probable cause. MRCP is a relatively new imaging technique in our country for the evaluation of obstructive jaundice and data from our part of the world is limited. It was introduced in $1991^{3}$.

The study is aimed at evaluating the role of MRCP in clinically suspected cases of obstructive jaundice. It tried to determine the diagnostic accuracy of MRCP in the detection of the cause of obstructive jaundice which helps in early and prompt treatment and limits the use of invasive endoscopic retrograde cholangiopancreatography (ERCP) as a treatment.

MRCP is a new modality which should be the first choice in the study of obstructive jaundice due to its non invasiveness and high accuracy. ${ }^{4-8}$

\section{MATERIALS AND METHODS}

Twenty patients with clinical \& biochemical features of obstructive jaundice who presented to the department of radiodiagnosis between October 2015 and September 2016 were enrolled in this study. This study was carried out at Susheela Tiwari Hospital, Government Medical College, Haldwani. Data collection regarding patient age, sex, address and specific imaging findings were obtained in each case and recorded on a special protocol form. All these patients were evaluated by Magnetic resonance imaging-Magnetic resonance cholangiopancreatography (MRI-
MRCP).

MRI Examination: The examination was done using Siemens Somatom 1.5T MRI machine. We checked the patients for any contraindications. No special preparation was needed. A body coil was used for the examination. Conventional MRI of the upper abdomen was done including $\mathrm{T} 1$ and $\mathrm{T} 2$ with or without fat suppression technique. I.V. contrast medium was given, if required (Gadolinium DTPA in a dose of $0.1 \mathrm{mmol} \mathrm{Kg}$ as a bolus).

Prior to Magnetic resonance cholangiopancreatography, the patient will be fasting for 4-6 hours and fully explained consent will be taken. During this period, patient is allowed to consume clear liquids, especially water. Magnetic resonance cholangiopancreatography will be performed using Siemens Somatom 1.5 Tesla MRI machine.

The Magnetic resonance cholangiopancreatography findings will be correlated with clinical history, examination, biochemical tests and surgical findings (if patient is operated)/ endoscopic retrograde cholangiopancreatography.

\section{RESULTS}

Eighty patients with clinical \& biochemical features of obstructive jaundice who presented to the department of radiodiagnosis were evaluated by MRI-MRCP.

\section{DETECTION OF DUCTAL DILATATION}

In our study, 20 patients were found to have ductal dilatation on MRI-MRCP. MRI-MRCP was able to diagnose ductal dilatation in all the cases.

Table 1: Detection of ductal dilatation

\begin{tabular}{|c|c|c|c|c|}
\hline 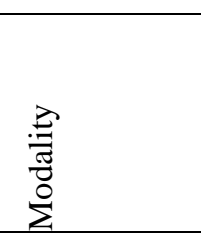 & 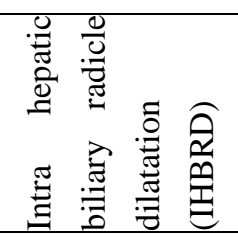 & 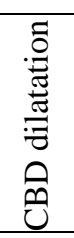 & 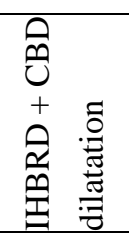 & 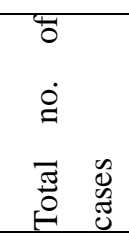 \\
\hline MRI-MRCP & 6 & 2 & 12 & 20 \\
\hline
\end{tabular}




\section{DETECTION OF LEVEL OF OBSTRUC- TION}

In our study, the most common level of obstruction was found to be at the distal common bile duct (CBD). MRCP was able to accurately detect the level of obstruction in all the patients.

Table 2: Detection of level of obstruction

\begin{tabular}{|l|c|c|}
\hline Level of Obstruction & No. of cases & $\begin{array}{c}\text { MRCP } \\
\text { (no. of cases) }\end{array}$ \\
\hline Porta hepatis & 5 & 5 \\
\hline Prox. CBD & 3 & 3 \\
\hline Distal CBD & 12 & 12 \\
\hline Total NO. of cases & 20 & 20 \\
\hline
\end{tabular}

\section{DETECTION OF CAUSE OF OBSTRUC-}

\section{TION}

Based on surgical findings (with histopathological confirmation) and / or ERCP findings, we found that the most common cause of obstructive jaundice in our study was tumors $(50 \%)$, followed

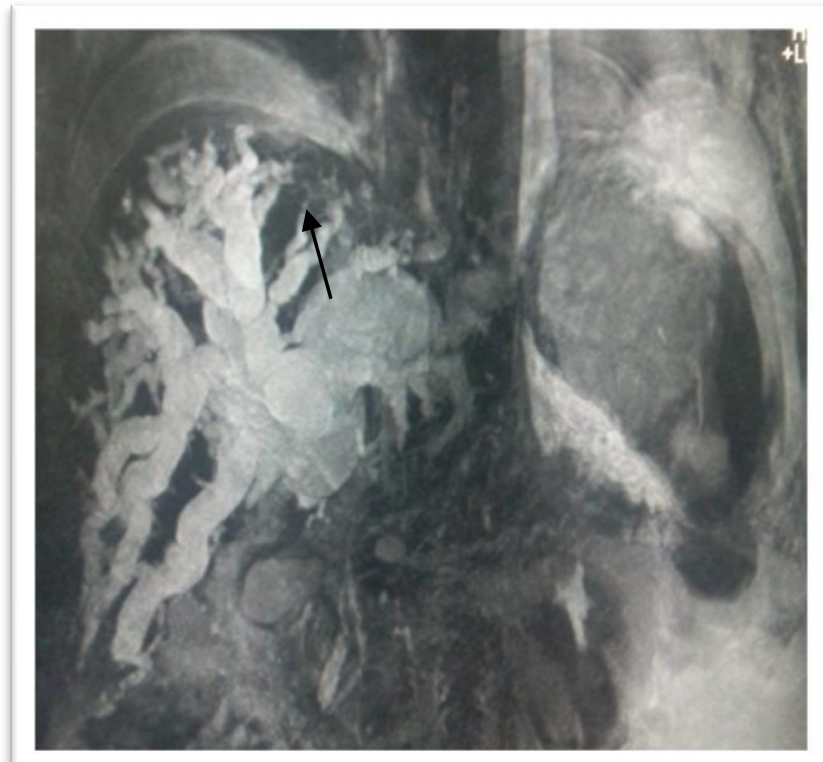

by choledocholithiasis (40\%), benign CBD strictures $(5 \%)$ and lymph nodal mass $(5 \%)$. The most common benign cause of obstructive jaundice is common bile duct calculi.

In our study, MRI-MRCP was accurately able to detect the cause of obstruction in 19 / 20 patients $(95 \%)$.

Table 3: Detection of cause of obstruction

\begin{tabular}{|l|c|c|}
\hline Cause of obstruction & No. of patients & MRCP \\
\hline CBD calculi & 8 & 8 \\
\hline Tumors & 10 & 9 \\
\hline Benign strictures & 1 & 1 \\
\hline Lymph nodal mass & 1 & 1 \\
\hline Total & 20 & 19 \\
\hline
\end{tabular}

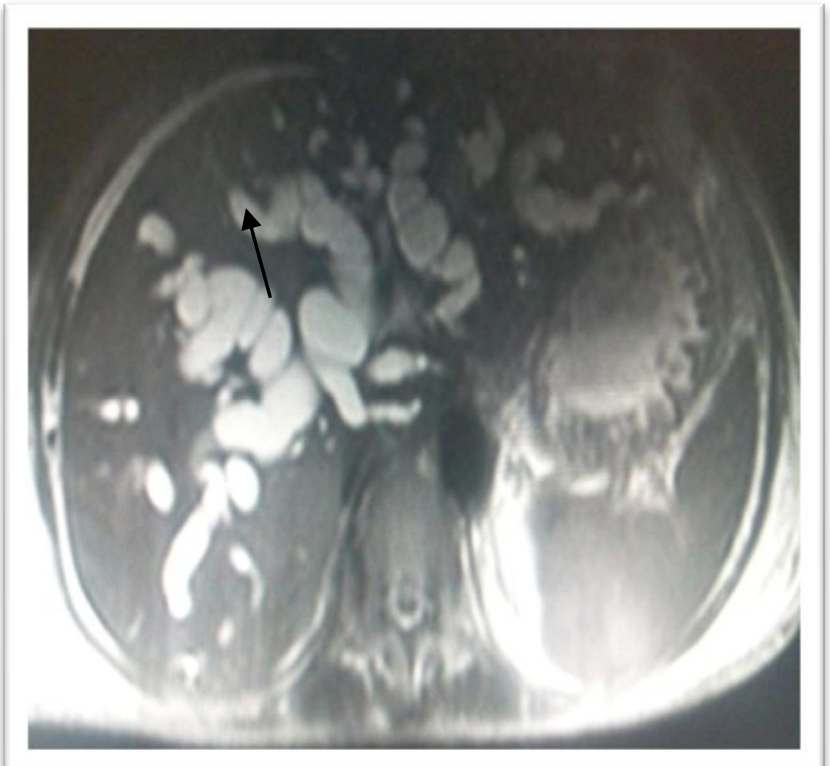

Figure 1 (A \& B) : Shows marked intrahepatic biliary radicle dilatation (black arrows) on coronal and axial T2 W MR images. 


\section{JMSCR Vol||05||Issue||03||Page 18310-18315||March}

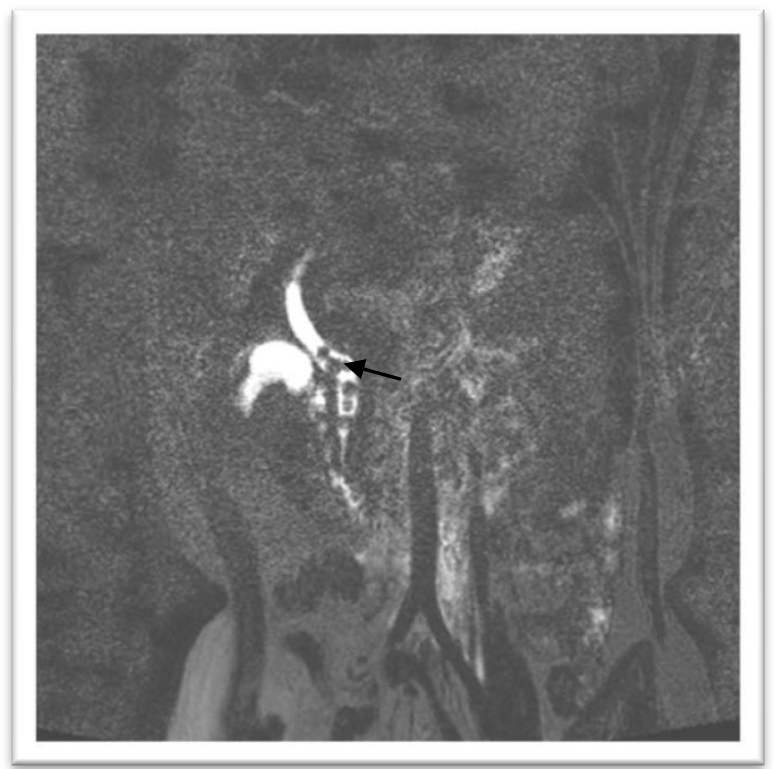

Figure 2 : Multiple common bile duct calculi (black arrow) as seen on MRCP image.

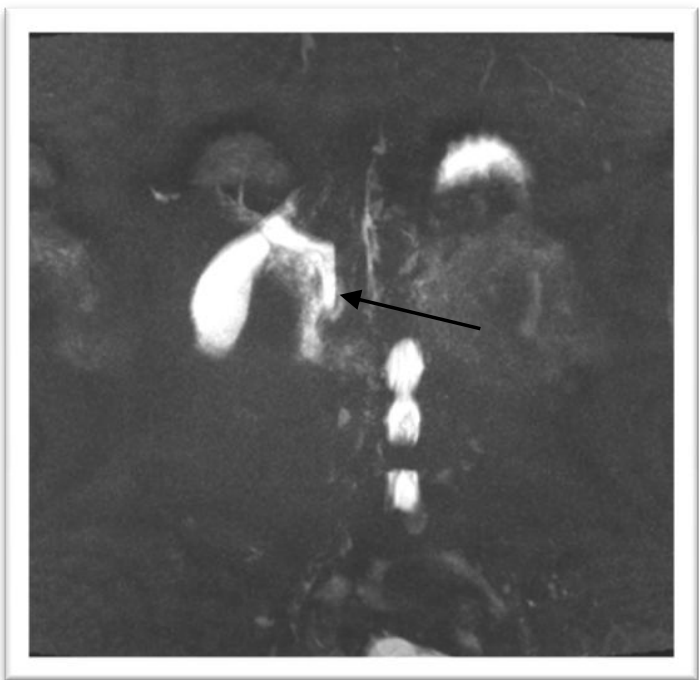

Figure 3 : Shows stricture of common bile duct in it's mid $1 / 3^{\text {rd }}$ (black arrow) as seen on MRCP image.

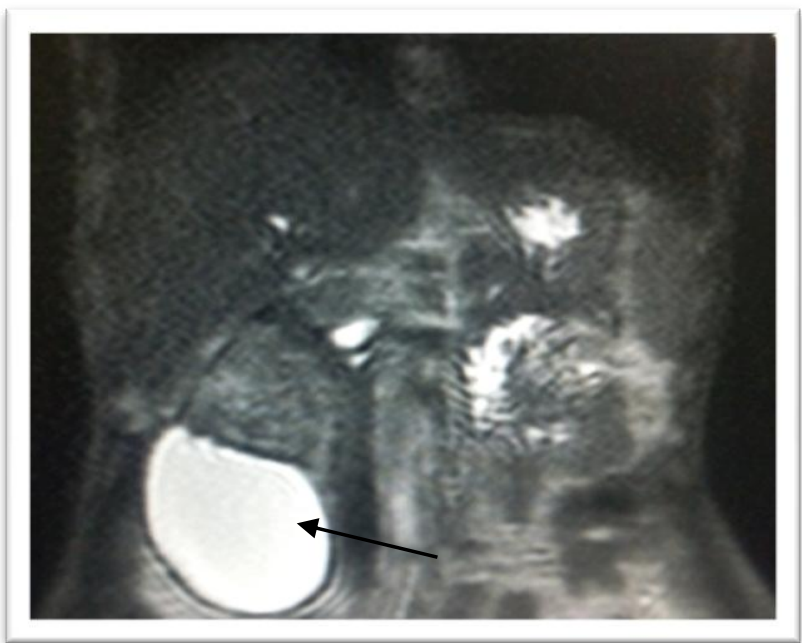

Figure 4 : Shows a gall bladder mass (black arrow) in the region of distal body and neck on coronal T2 W MR image. 


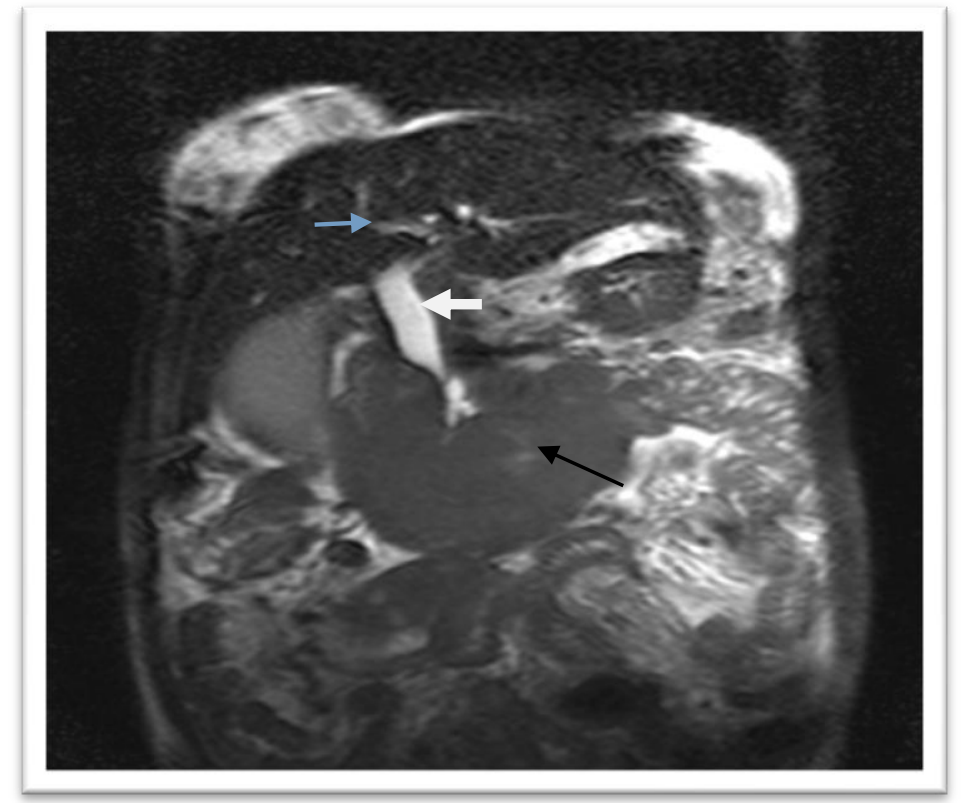

Figure 5: Coronal T2 W MR image shows a large conglomerated lymph nodal mass (black arrows) in the peripancreatic region, causing extrinsic compression of the distal common bile duct (white arrow), with resultant dilatation of proximal common bile duct and intrahepatic biliary radicle dilatation (blue arrow).

\section{DISCUSSION}

Jaundice is a common problem in both medical and surgical practice. Its cause can often be correctly anticipated clinically, but usually biochemical and radiological imaging investigations are required for confirmation. The main goals of any imaging procedure in obstructive jaundice are to confirm the presence of obstruction, its location, extent, probable cause.

In our study, the accuracy of magnetic resonance cholangiopancreatography in the detection of ductal dilatation, level of obstruction and cause of obstruction was $100 \%, 100 \%$ and $95 \%$ respectively. The results obtained in our study are comparable to the results obtained by Safa $\mathrm{Al}-$ Obaidi et al ${ }^{9}$ in their study. In our study, we found the distal common bile duct to be the most common site of obstruction (56.25\%). Similar observation was reported by Bhavesh Goyani et al 10 and Safa Al- Obaidi et al $^{9}$ in their studies. V Upadhyaya et al ${ }^{11}$ in their study found the intrapancreatic common bile duct to be the most common site of obstruction (38\%), followed by the suprapancreatic common bile duct (34\%) and the porta hepatis (28\%).

The most common cause of obstruction in our study was pancreatico-biliary tumors (47.5\%), followed by choledocholithiasis (40\%). A similar observation was made by V Upadhyaya et al ${ }^{11}$ and Rajneesh Madhok et al ${ }^{12}$ in their studies. However, Mubarak Ali et al ${ }^{13}$ (at Karachi) and Manohar Singh Rathore et al ${ }^{2}$ (at Delhi) observed that choledocholithiasis was the most common cause of obstructive jaundice.

\section{CONCLUSION}

Differentiation of obstructive jaundice from medical jaundice is imperative for the management of the patient.

From this study, it is concluded that Magnetic resonance imaging - magnetic resonance cholangiopancreatography (MRI-MRCP) is a highly sensitive non-invasive technique in the detection of the presence of biliary obstruction in addition to defining its level and cause. The accuracy of magnetic resonance cholangiopancreatography and its non-invasiveness have considerably restricted the diagnostic role of 
Endoscopic retrograde cholangiopancreatography (ERCP).

The advantages of MRCP were found to be:

High-resolution images of the biliary tree.

Acquisition of diagnostic images both above and below the level of obstruction.

Three-dimensional (3D) image of the biliary tree, which can help in treatment planning.

It can be used in obese patients and those patients who are poor ultrasonography candidates due to technical factors or limited field of view as in postoperative patients.

\section{REFERENCES}

1. Scharsshmidt GF, Goldberg HI, Schmid R. Approach to the patient with cholestatic -jaundice. N Engl J Med. 1983; 308(25): 1515-9.

2. Rathore et al.; Comparative Study to Assess Diagnostic Reliability of Ultrasound and 3T-MRCP in Patient of Obstructive Jaundice. BJMMR. 2015: 8(11): 919-930.

3. Glockner JF. Hepatobiliary MRI: current concepts and controversies. J Magn Reson Imaging. 2007; 25(4): 681-95 [PubMed].

4. Kaltenthaler EC, Walters SJ, Chilott J, Blakerborough A, Vergel YB, Thomas S. MRCP compared to diagnostic ERCP for diagnosis when biliary obstruction is suspected: a systematic review. BMC Med Imaging 2006; 6: 9.

5. Di Cesare E, Puglielli E, Michelini O, Pistoi MA, Lombardi L, Rossi M, et al. Malignant obstructive jaundice: comparison of MRCP and ERCP in evaluation of distal lesions. Radiol Med 2003; 105: 445-53.

6. Hekimoglu K, Ustundag Y, Dusak A, Erdem Z, Karademir B, Aydemi S, et al.
MRCP vs ERCP in the evaluation of biliary pathologies: review of current literature. J Dig Dis 2008; 9: 162-9.

7. Iannicceli E, Sessa A, Argnani L, Galluzzo A, David V. [Role of MR Cholangiopanc -reatography in the evaluation of biliary disease]. Clin Ter 2006; 157: 425-9.

8. Park DH, Kim MH, Lee SS, Lee SK, Kim KP, Han JM, et al. Accuracy of magnetic resonance cholangio-pancreatography for locating hepatolithiasis and detecting accompanying biliary strictures. Endoscopy 2004; 36: 987-92.

9. Safa Al-Obaidi, Mohammed Ridha Alwan Al-Hilli et al. The Role of Ultrasound and Magnetic Resonance Imaging in the Diagnosis of Obstructive Jaundice. The Iraqi Post Graduate Medical Journal 2007: 6 ( 11).

10. Bhavesh Goyani, Bhagvati Ukani et al. Ultrasonography and magnetic resonance cholangiopancreatography correlation in patients with obstructive jaundice. IJMSPH 2015 : 4 (7); 1010-1016.

11. Upadhyaya V, Upadhyaya DN, Ansari MA, Shukla VK. Comparative Assessment Of Imaging Modalities In Biliary Obstruction. Ind J Radiol Imag.2006; 16(4): 577-82.

12. Rajneesh Madhok, Swinki Rastogi. Role of 3.0 Tesla Magnetic Resonance Cholangiopancreatography in Obstructive Jaundice with Cyto/Histopathological or Surgical Correlation. IJSS 2015; 3( 2).

13. Mubarak Ali, Ishtiaq Ahmed et al. Diagnostic accuracy of magnetic resonance cholangio-pancreatography in evaluation of obstructive jaundice. JPMA 2012; 62: 105. 